\title{
A Practical Estimating Method of the Dose-Response Curve between Inoculum Density of Plasmodiophora brassicae and the Disease Severity for Long-term IPM Strategies
}

\author{
Seiya TSUSHIMA ${ }^{1}$, Hiroharu MURAKAMI ${ }^{2 *}$, Takayuki AKIMOTO \\ Mitsuhiko KATAHIRA ${ }^{4}$, Yukiko KUROYANAGI ${ }^{2}$ and \\ Yoshihiro SHISHIDO ${ }^{4}$
}

Department of Integrated Research for Agriculture, National Agricultural Research Center for Tohoku Region, National Agriculture and Food Research Organization (NARO)

(Fukushima, Fukushima 960-2156, Japan)

\begin{abstract}
A model was developed to evaluate long-term temporal changes in disease severity (DS) of the clubroot disease of Chinese cabbage in the field with various management strategies. The model consists of a dose-response curve (DRC) of resting spore (RS) density of Plasmodiophora brassicae and DS, and the rate of RS density change due to leafy daikon cropping as a decoy plant, plowing the clubbed roots in the soil after harvest, and natural reduction. The DRC was estimated by greenhouse experiments. Three DRCs were estimated by experiments carried out in 1995-1997. The DRC in 1996 predicted the highest DS at all RS densities and hence was considered to represent a conducive condition, and that in 1997 predicted the lowest DS and was considered to represent a suppressive condition, respectively. Field data for three years (1996-1998) fell into an area surrounded by both DRC. This result confirmed the validity of the DRCs. Also the model was validated by field experiments where leafy daikon was cultivated before planting Chinese cabbage. The usefulness of the model for longterm prediction of temporal changes of DS was discussed.
\end{abstract}

Discipline: Plant disease

Additional key words: clubroot, crop rotation, disease control, population dynamics

\section{Introduction}

Clubroot disease caused by Plasmodiophora brassicae brings serious yield loss in crucifers due to the increase in population density of the pathogen with their continuous monoculture ${ }^{8}$. Although its control exclusively depends on the use of chemicals, public concerns for their environmental risks have grown. Introduction of the concept of integrated pest management (IPM) including a combination of several alternative control measures is necessary.

Recently, we demonstrated ${ }^{5,6}$ that cultivation of leafy daikon (Raphanus sativus var. longipinnatus) reduced density of resting spores (RS) of the pathogen in the soil by 75 and $95 \%$ compared with the control without leafy daikon in greenhouse and field experiments, respectively. Though leafy daikon promotes the germination of RS in the soil, the pathogen cannot multiply in leafy daikon plants and subsequently dies out. As a result, the RS density of the pathogen in the soil decreases after cropping leafy daikon. The disease severity (DS) in succeeding

\footnotetext{
Present address:

${ }^{1}$ National Institute for Agro-Environmental Sciences (NIAES) (Tsukuba, Ibaraki 305-8604, Japan)

${ }^{2}$ National Institute of Vegetable and Tea Science, NARO (Tsukuba, Ibaraki 305-8666, Japan)

${ }^{3}$ Unzen Station, National Center for Seeds and Seedlings (Unzen, Nagasaki 859-1211, Japan)

${ }^{4}$ Faculty of Agriculture, Yamagata University (Tsuruoka, Yamagata 997-8555, Japan)

*Corresponding author: e-mail hmura@affrc.go.jp

Received 23 January 2009; accepted 3 March 2010.
} 
Chinese cabbage plants also generally decreased with the RS density. Thus, leafy daikon functions as a decoy plant $t^{4}$ and suggests the usefulness of leafy daikon cropping as one of the alternative control measures in IPM. However, leafy daikon cropping was less effective in soil with high RS densities. This fact could be consistent with this control measure if we assume that the relationship between RS density and DS obeys the dose-response curve (DRC), and that cultivation of leafy daikon simply reduces RS density. That is, when the density is relatively high and considered to be the stationary phase of the sigmoid DRC, the DS would not sensitively respond to the change of RS density. This indicates that estimating DRC and the initial RS density in the target fields are critical for predicting whether introduction of leafy daikon in the cropping system is effective on suppressing clubroot disease. Meanwhile, RS density in the soil has been known to increase with plowing the clubbed roots after harvest, which may cause the disease occurrence to be more severe ${ }^{3}$. To evaluate the effect of the plowing practice on the DS, the use of the DRC concept could be also helpful.

Mathematical modeling of a plant pathosystem is a useful tool to aid in understanding the effect of various factors on disease occurrence and exploring better management strategies. For the clubroot disease of crucifer, Amano et al. ${ }^{1}$ introduced a mathematical model to relate RS density in the soil to DS. Their model arises from the DRC. If the effects of a decoy plant cropping and plowing clubbed roots on RS density are incorporated into the DRC model, comprehensive understanding of long-term disease management strategies could be explored easily. However, the parameters of the DRC have to be individually estimated for each soil type, soil $\mathrm{pH}$, cultivar of crop, and so on. Moreover, they suggested that the parameters have to be estimated even for each plot within a soil type due to heterogeneity of various factors such as soil $\mathrm{pH}$. Obviously, it is time-consuming and laborious. This implies that estimation of RS density based on the DRC could not be always applicable from a practical point of view. For this reason, a simple method to estimate DRC of a target cultivation system is necessary for the effective use of the model.

We report here a simple and rapid estimating method of DRC of the clubroot disease of Chinese cabbage. Then we introduce a mathematical model which consists of DRC and three factors affecting RS density of the pathogen including decoy plant cropping, plowing clubbed roots and natural reduction. Finally, we demonstrate the model by predicting the temporal changes of the RS density and DS in a crop rotation system (leafy daikon - Chinese cabbage).

\section{Materials and methods}

\section{Measurement of the resting spore density in soil and assessment of disease severity}

RS density in the soil was measured fluorescent-microscopically by staining soil extraction with calcofluor white M2R (SIGMA) as previously described ${ }^{5}$. DS was assessed 35 days after sowing in greenhouse experiments and on harvest in field experiments, respectively. Each Chinese cabbage plant assessed was rated into 4 categories ( 0 to 3 ) according to Dixon et al. ${ }^{2}$ : category 0 , not clubbed; category 1, only lateral roots clubbed; category 2 , less than half of the tap root clubbed; category 3 , more than half of the tap root clubbed. DS was calculated as follows:

$$
\mathrm{DS}=\left(1 \times \mathrm{n}_{1}+2 \times \mathrm{n}_{2}+3 \times \mathrm{n}_{3}\right) /(3 \times \mathrm{N}) \times 100
$$

where, $\mathrm{N}$ is the total number of plants and $\mathrm{n}_{\mathrm{i}}$ is the number of plants classified as category i. DS was considered to be a continuous variable for approximation, while these categories were considered to be an ordinal rating scale ${ }^{11}$.

\section{Modeling}

The dose - response relationship was assumed to be described according to the equation ${ }^{9}$ below:

$$
Y=\mathrm{k}(1-\exp [-\mathrm{w} x])
$$

in which $Y$ and $x$ are DS and RS density in the soil and $\mathrm{k}$ and $\mathrm{w}$ are the parameters, respectively.

The effect of leafy daikon cropping on reduction of $\mathrm{RS}$ density in the soil is shown as the equation below:

$$
X_{t+1}=(1-\mathrm{m}) X_{t}
$$

where $\mathrm{m}$ is the proportion of reduction in RS density due to each leafy daikon cropping, $X_{t}$ is $\mathrm{RS}$ density at $\mathrm{t}$ and $\mathrm{t}$ is the starting point of each cropping, respectively.

The effect of plowing clubbed roots after cultivating Chinese cabbage on RS density is shown as the equation below:

$$
X_{t+1}=X_{t}+\mathrm{f}_{\mathrm{p}}\left(X_{t}\right)
$$

where $f_{p}$ is the increase in RS density due to plowing clubbed roots.

The crop-to-crop change in RS density is shown as the equation below:

$$
X_{t+1}=(1-\mathrm{q}-\mathrm{m}) X_{t}+\mathrm{f}_{\mathrm{p}}\left(X_{t}\right)
$$


where $\mathrm{q}$ is the natural reduction per crop (half year).

\section{Estimating parameters of the DRC}

Haplic Andosol (HA-soil) collected from a field at National Agricultural Research Center for Tohoku Region (NARCT), Fukushima campus was used in greenhouse experiments. DRCs showing the relationship between RS density in HA-soil and DS of Chinese cabbage (Brassica rapa var. pekinensis, cv. Shin-azuma) were obtained in 1995, 1996 and 1997 as described by Murakami et al.?

\section{Field experiments}

To validate if DRCs estimated from greenhouse experiments could apply to the data from field experiments in NARCT, Chinese cabbages (cv. Shin-azuma and cv. Victoria) were planted in naturally infested fields with different densities of RSs in 1996, 1997 and 1998. The experimental design used was a randomized complete block design with three replicates. Each plot consisted of a $2.0 \times 8.0 \mathrm{~m}$ rectangular field. RS density in soil was measured before cultivating Chinese cabbage and DS of Chinese cabbage was investigated on harvest.

Clubbed roots were recovered randomly from 18 naturally infested fields in NARCT with different DS and the amounts of RSs per clubbed root were determined by microscopy observation. Then the amount of RSs introduced into the field was estimated using the data from naturally infested fields with various DSs.

Leafy daikon (breeding line CR-1) was sown at a rate of $75 \mathrm{ml}$ seeds per plot $(1.6 \times 7.5 \mathrm{~m})$ in the infested field at NARCT as the preceding crop and grown for 1.5 months. Then, they were plowed into soil by machinery and the field fallowed for 1.5 months to promote decomposition. Then, Chinese cabbage was sown with $80 \mathrm{~cm}$ row spacing and $30 \mathrm{~cm}$ intervals between plants in August and grown for about 2 months. Experiments were carried out for 3 years and each plot had triplicate replications. All clubbed roots were plowed by machinery or dug up by hand from plots just after the DS was determined at harvest. Two soil samples consisting of 5 subsamples (depth: 0 to $10 \mathrm{~cm}$ ) were collected from each treatment plot and the RS density in the soil was determined.

\section{Model performance}

The reduction in RS density due to leafy daikon cropping was simulated using the model (Eq. iv), assuming the proportion of reduction in RS density due to leafy daikon cropping (m) as 0.7 based on the previously obtained data from other greenhouse and field experiments ${ }^{5}$. The natural reduction in RS density during each cropping (half year) was assumed to be 0.5 , based on a preliminary experiment (data not shown).

\section{Results}

\section{Estimation of the parameters from the results of greenhouse experiments}

The patterns of the dose-response relationship varied among the 3 experiments. DS in 1996 tended to be higher at all inoculum densities than in 1995 and 1997. The curves fitting these relationships were obtained by changing parameters $\mathrm{k}$ and $\mathrm{w}$ of the modified one-hit model as follows, $Y=\mathrm{k} \times(1-\exp [-\mathrm{w} x])$. The upper curve fit well the results of the 1996 experiment when parameters $\mathrm{k}$ and $\mathrm{w}$ were 99.8 and $9.20 \times 10^{-4}$, respectively, and the lower curve fit those of 1995 and 1997 experiment when parameters $\mathrm{k}$ and $\mathrm{w}$ were 94.2 and $0.95 \times 10^{-4}$, respectively (Fig. 1).

\section{Validation of the DRC by field experiments}

To validate the DRCs estimated from greenhouse experiments, the data obtained from field experiments in 1996, 1997 and 1998 were plotted on a graph and graphically compared (Fig. 2). Twenty three of the data points fell in the area between DRCs from the greenhouse experiments described above. It implies that these DRCs were useful to describe the relationship between RS density and DS in naturally infested fields (Fig. 2),

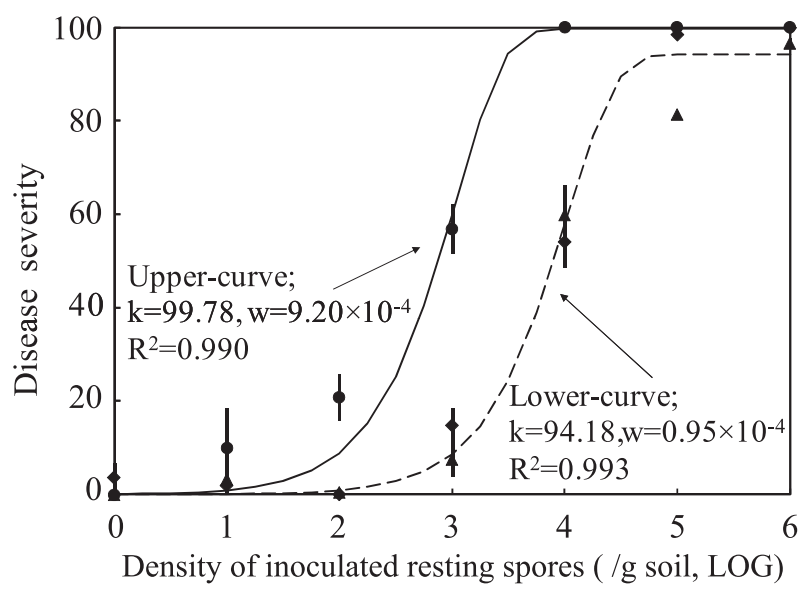

Fig. 1. Dose-response relationship of the clubroot disease estimated by greenhouse experiments carried out in 1995, 1996 and 1997

Dose-response relationship is represented as the equation; $Y=\mathrm{k}(1-\exp [-\mathrm{w} x])$, in which $Y$ and $x$ are DS and RS density in the soil and $\mathrm{k}$ and $\mathrm{w}$ are the parameters, respectively. The upper- and lowercurves represent a disease conductive case and a disease suppressive case. Bars show SE.

$\checkmark$ : 1995data, : 1996data, $\boldsymbol{\Delta}$ : 1997data. 


\section{Estimation of the amount of RSs by plowing clubbed roots}

The mean density of RS within individual clubbed roots with category 1, 2 and 3 were $9.2 \pm 0.21$ (mean log value $\pm \mathrm{SE}$ ), $9.6 \pm 0.14$ and $10.2 \pm 0.12$, respectively. The RS density in infested fields could be estimated using three values assuming that clubbed roots were uniformly distributed in the range of $20 \mathrm{~cm}$ deep in an area of 2,400 $\mathrm{cm}^{2}(30 \times 80 \mathrm{~cm})$ according to the cultivation system in these fields. From these data, the relationship between the DS and the amount of RS introduced into a field by plowing clubbed roots was shown as:

$$
Y=0.5448 \ln (X)+3.1136, R^{2}=0.954,
$$

where $Y$ was the amount of RS ( $\mathrm{g}^{-1}$ soil, log value) introduced into a field by plowing clubbed roots and $X$ was the DS of Chinese cabbage plants in the field (Fig. 3).

\section{Model performance}

To evaluate the model performance, the RS density and DS assessed in the fields, where the clubbed roots were removed, were compared with those predicted by the model (Fig. 4, A and B). These comparisons showed that the RS density simply decreased from 1997 to 2000 . The result did not contradict the RS density determined by direct measurement from the field soil samples (Fig. 4, A). On the other hand, the model predicted no decrease of DS based on the upper DRC scenario (the most pessimistic case), while a drastic decrease was predicted based

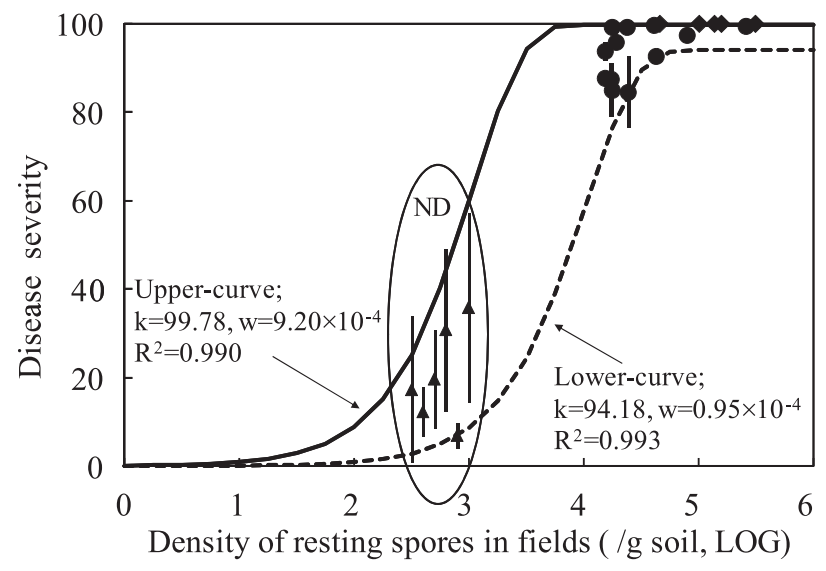

Fig. 2. Graphic validation of the dose response curves estimated by greenhouse experiments using field experiment data

Circle shows the fields in which RS was not detected (detection limit; $<10^{4}$ resting spores $\mathrm{g}^{-1}$ soil). Bars show SE.

: 1996-1998 field (A), $\bullet$ : 1997 field (B), A : 1998 field (C). on the lower DRC scenario (the most optimistic case). The result from field experiments was consistent with the pessimistic case (Fig. 4, B).

When leafy daikon had been cultivated before Chinese cabbage whose clubbed roots were plowed, the model showed no decrease in RS density over 3 years, though RS density occasionally decreased after leafy daikon. The result was consistent with the data observed in a naturally infested field (Fig. 5, A). Adding to that, the temporal changes of DS predicted by the model were consistent with those observed in the fields (Fig. 5, B). These results strongly suggested the usefulness of the model parameterized by data from greenhouse experiments.

The long-term simulation was performed to examine (1) the effect of the initial RS density on the subsequent level of the RS density and DS in continuous monoculture of Chinese cabbage where the clubbed roots were plowed after harvest (Fig. 6, A-D), and (2) the effect of the initial RS density and introduction of leafy daikon cropping into a crop rotation system where the clubbed roots were plowed after harvest (Fig. 7, A-D). The simulation indicated that crop rotation (Chinese cabbage and leafy daikon) could be effective in the optimistic cases of the scenario, while continuous monoculture of Chinese cabbage causes severe disease consequences, except the cases that the initial RS densities are very low.

\section{Discussion}

In Japan, many cruciferous crops have been consecutively cultivated, and hence have often suffered from clubroot disease. Assessing the density of resting spores (RS) of the pathogen in the soil is essential to evaluate ef-

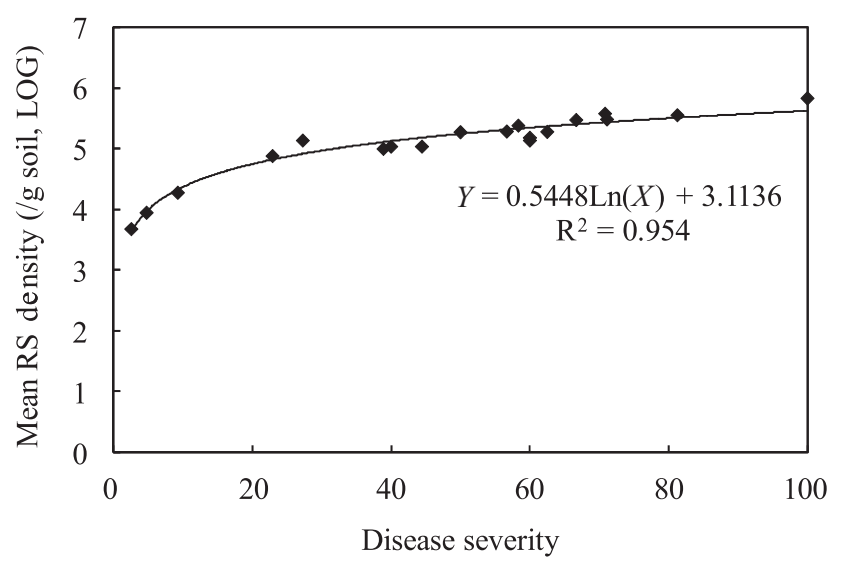

Fig. 3. Estimated RS density of Plasmodiophora brassicae in fields with plowing of Chinese cabbage clubbed roots by the disease severity

$Y$ and $X$ are RS density in the soil and DS, respectively. 
fectiveness of various control measures, because efficacy of the measures depends on the density of RS in the soil, as well as soil condition, crop susceptibility and so on? In this paper, first we reported the usefulness of DRC obtained simply and rapidly from greenhouse experiments. Subsequently we validated the DRC model and the integrated model for planning IPM strategies, which consists of the DRC model and other components to regulate RS density.

Because DRC was considerably regulated by environmental conditions including soil characteristics, kind of crop and virulence of the pathogen ${ }^{7}$, DRC should have been individually estimated for each field. However, since estimating DRC from field experiments is laborious and time-consuming, DRCs were alternatively estimated from greenhouse experiments in this study. Two DRCs were chosen from three years' experiments (1995-1997) as the upper and lower DRCs (Fig. 1), because estimated DRCs varied even under the greenhouse conditions. To validate the model, data obtained from three years of field observations (1996-1998) were plotted in Fig. 2. This procedure revealed that most of the observed data points were distributed within the area surrounded by these two DRCs, while values of the data obtained from naturally infested fields varied year by year. This clearly indicates that the DRC model estimated from greenhouse experi-
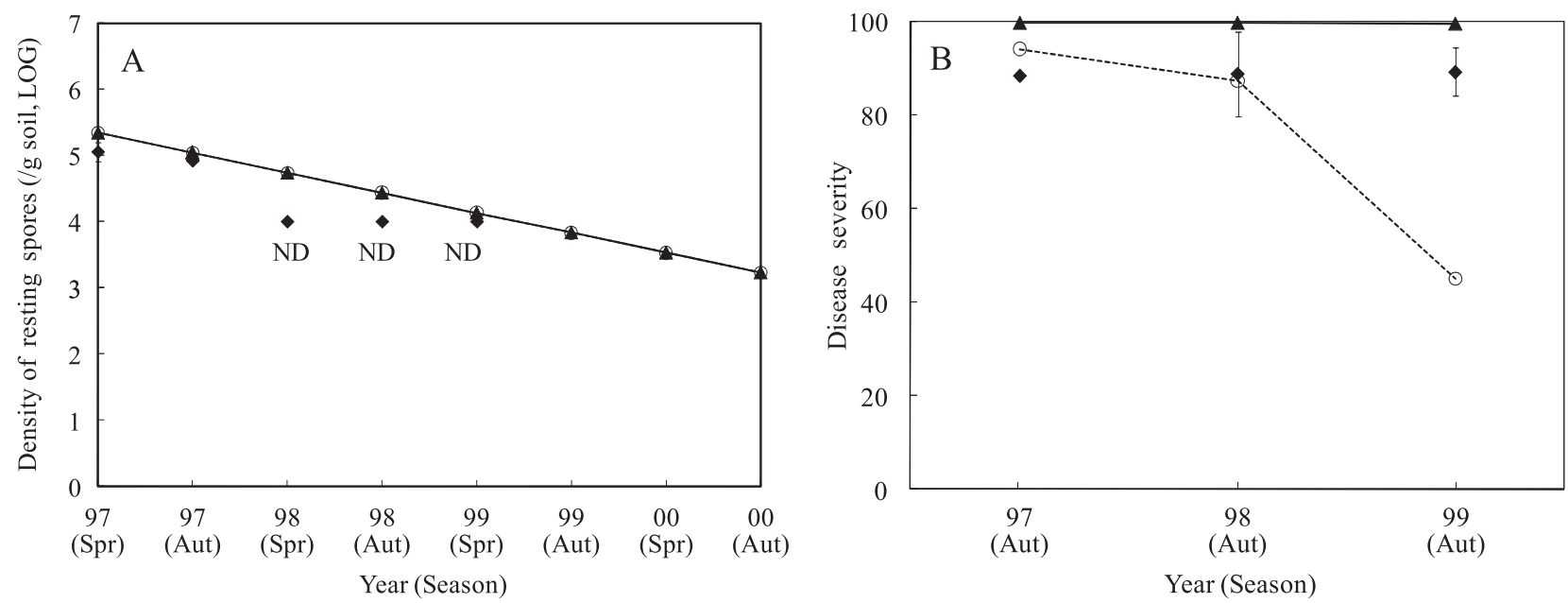

Fig. 4. Graphic validation of the DRC model including a removal/plowing process of the Chinese cabbage clubbed roots using data from fields where the roots were removed from the soil

Yearly change in the density of resting spores (A) and in the disease severity of clubroot (B). ND data are plotted on the line of detection limit of RS in soil $\left(<10^{4}\right.$ resting spores $\mathrm{g}^{-1}$ soil). Bars show SE.

- : Based on Upper DRC curve, $-\odot-$ : Based on Lower DRC curve, $\diamond$ : Field data.
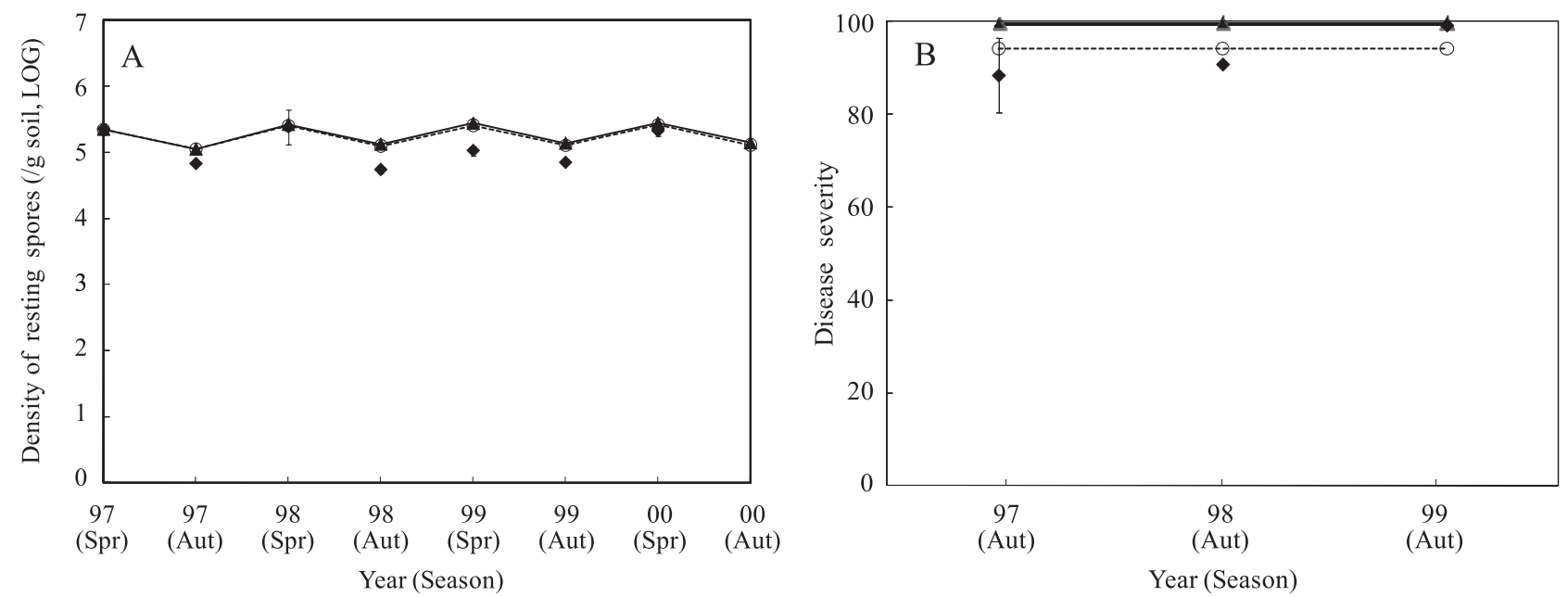

Fig. 5. Graphic validation of the DRC model including a removal/plowing process of the Chinese cabbage clubbed roots using data from fields where the roots were plowed into the soil

Yearly change in the density of resting spores (A) and in the disease severity of clubroot (B). Bars show SE.

- : Based on Upper DRC curve, -- $\odot--$ : Based on Lower DRC curve, $\diamond$ : Field data. 

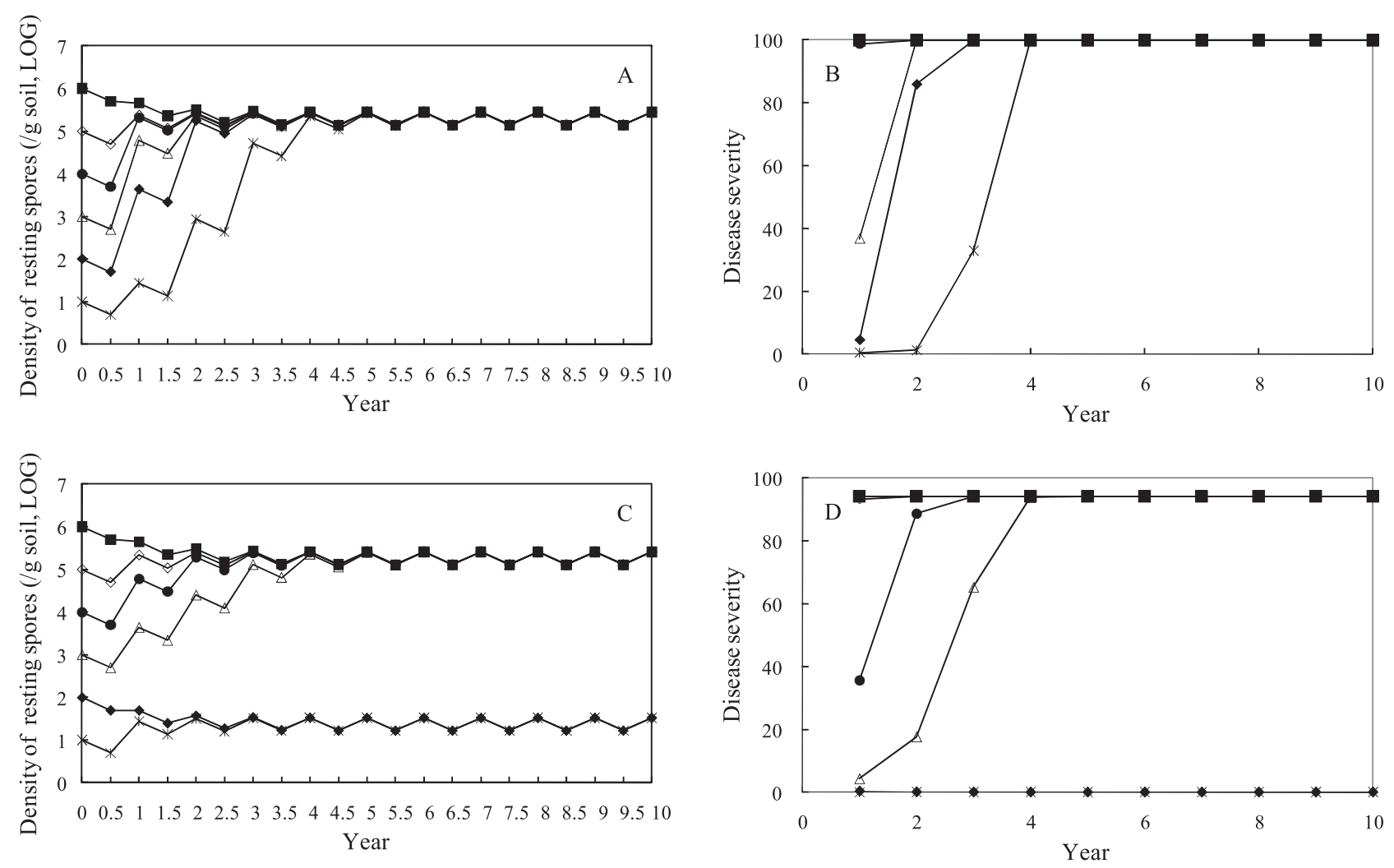

Fig. 6. Long-term simulation by the DRC model at various initial RS densities of Plasmodiophora brassicae in the soil in a monoculture field of Chinese cabbage where the clubbed roots were plowed after harvest

$\mathrm{A}$ and $\mathrm{C}$, yearly change in the density of resting spores by simulation using 'upper curve' and 'lower curve', respectively; $\mathrm{B}$ and $\mathrm{D}$, yearly change in disease severity of clubroot by simulation using 'upper curve' and 'lower curve', respectively. Inoculum density, $\mathbf{\square}: 10^{6} / \mathrm{g}, \diamond: 10^{5} / \mathrm{g}, \bigcirc: 10^{4} / \mathrm{g}, \triangle: 10^{3} / \mathrm{g}, \diamond: 10^{2} / \mathrm{g}, *: 10^{1} / \mathrm{g}$.

ments are valid, and that the DRC model could be consequently applicable to analyze the relationship between the density of RS and disease severity (DS). Ultimately, the upper DRC (a pessimistic case) may represent a conducive case to the disease, while the lower DRC (an optimistic case) may be a suppressive case, respectively.

Subsequently, we constructed a mathematical model to integrate 4 components to simulate temporal changes of RS density and the disease severity in various rotation cropping systems. The model consisted of (1) the initial inoculums of RS density in the soil, (2) the reduction rate of RS density by cropping a decoy plant, (3) the increase rate of RS density by plowing clubbed roots, and (4) the natural reduction rate of the RS density. The results of long-term prediction with the model using 0.5 as the value of natural reduction (q) revealed that RS density in soil was less than $10^{6}$ spores $\mathrm{g}^{-1}$ soil (Fig. 6). The RS densities in the fields that we investigated in Japan were usually below $10^{6}$ spores $\mathrm{g}^{-1}$ soil (data not shown). In addition, the simulation using 0.5 as the proportion of reduction in RS density (m) almost corresponded with the results of Wallenhammer ${ }^{10}$ that the level of infection declined to be- low the detection threshold after 17.3 years without Brassica crops. This suggested that the value, 0.5 was suitable as the proportion of natural reduction (q).

This integrated model was validated in the leafy daikon-Chinese cabbage rotation system by comparing data observed in the field and the behavior of the model with corresponding parameters. That is, RS density observed in the field where clubbed roots of Chinese cabbage had been removed fitted fairly well the data predicted by the model (Fig. 4, A). Predicted DS varied with DRCs assumed in the model, while observed DS were distributed within the area surrounded by these two DRCs (Fig. 4, B). Both RS density and DS observed in the field where clubbed roots had been plowed were coincident with predicted values (Fig. 5). However, the result suggests that cultivation of a decoy plant may not be effective if clubbed roots were plowed in the soil in the cropping system. All of these results evidently showed that this integrated model is practically valid.

Now that the integrated model was validated as above, the model can be used for long-term prediction of the RS density and disease severity. To demonstrate the 

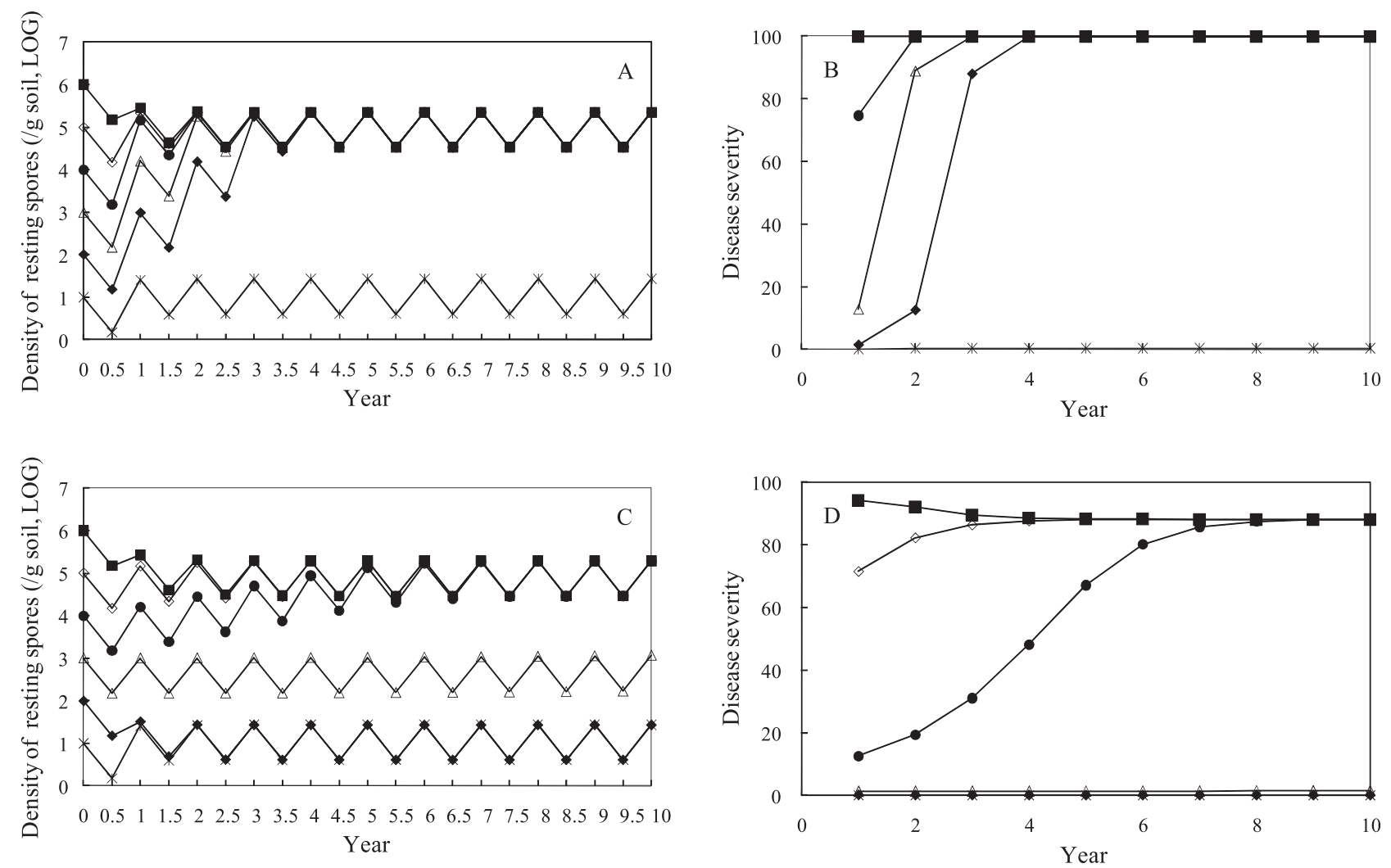

Fig. 7. Long-term simulation by the DRC model at various initial RS densities of Plasmodiophora brassicae in the soil of a Chinese cabbage-leafy daikon rotation field where clubbed roots were plowed after harvest

$\mathrm{A}$ and $\mathrm{C}$, yearly change in the density of resting spores by simulation using 'upper curve' and 'lower curve', respectively; $\mathrm{B}$ and $\mathrm{D}$, yearly change in disease severity of clubroot by simulation using 'upper curve' and 'lower curve', respectively. Inoculum density, $\mathbf{\square}: 10^{6} / \mathrm{g}, \diamond: 10^{5} / \mathrm{g}, \bigcirc: 10^{4} / \mathrm{g}, \triangle: 10^{3} / \mathrm{g}, \diamond: 10^{2} / \mathrm{g}, *: 10^{1} / \mathrm{g}$.

potential of the model as an aid in planning IPM strategies, several sensitivity analyses were carried out. Assuming a monoculture cropping system of Chinese cabbage, the model showed that RS density in the soil would increase to the level of $10^{5}-10^{6}$ spores $\mathrm{g}^{-1}$ soil except in the case of low initial RS density (Fig. 6, A and C). In this case, the predicted disease severity showed similar tendencies to the RS density (Fig. 6, B and D). Assuming a decoy plant-Chinese cabbage rotation system, where the clubbed roots of Chinese cabbage were plowed into the soil, the model using the upper DRC (pessimistic case) showed that RS density would converge on the level of $10^{4}-10^{5}$ spores $\mathrm{g}^{-1}$ soil within 3 years except in the case of very low initial RS density, where RS density would decrease after decoy plant cropping and increase after plowing clubbed roots in the soil (Fig. 7, A). In this case, the disease severity gradually increased to the maximum severity (100) within 4 years (Fig. 7, B). However, the model using the lower DRC (optimistic case) showed that RS density would remain at the level of $10^{1}-10^{2}$ spores $\mathrm{g}^{-1}$ soil and the disease severity declined to zero when the initial RS density was assumed to be less than $10^{2}$ spores $\mathrm{g}^{-1}$ soil (Fig. 7, C and D). That is, yearly interruptive cropping of a decoy plant would not effectively decrease RS density in the pessimistic case representing a disease conductive soil, while in the optimistic case representing a disease suppressive soil the cropping would be successful in the condition of low initial RS density. Thus, simulation using the model should be very useful to support a decision whether or not to introduce a decoy plant into the concerned cropping system.

Overall, these results showed that the mathematical model, which integrated a DRC model and sub-models to evaluate temporal changes of RS density, was validated by comparing observed data in the fields and predicted ones by the model output. This clearly indicated that this model can be applied to support decision-making in planning strategies for cropping systems based on IPM perspectives.

\section{Acknowledgments}

We thank Dr. N. Matsumoto (National Agricultural Research Center for Hokkaido Region, NARO, Japan) for 
critical reading of the manuscript, and Dr. K. Yamamura (NIAES) and Dr. S. Miyai (National Agricultural Research Center, NARO, Japan) for suggestions on a mathematical model.

\section{References}

1. Amano, T. et al. (1995) Development of decision support system for clubroot disease control in cabbage production based on long term prediction. Nogyo kenkyu senta hokoku (Bull. Natl. Agric. Res. Cent.), 24, 1-40 [In Japanese with English summary].

2. Dixon, G. R. \& Robinson, D. L. (1986) The susceptibility of Brassica oleracea cultivars to Plasmodiophora brassicae (clubroot). Plant Pathology, 35, 101-107.

3. Ikegami, H. (1992) Ecology of Plasmodiophora brassicae and control of clubroot disease. Tsuchi to biseibutsu (Soil Microorganisms), 39, 1-10 [In Japanese].

4. Macfarlane, I. (1952) Factors affecting the survival of Plasmodiophora brassicae Wor. in the soil and its assessment by a host test. Ann. Appl. Biol., 39, 239-256.

5. Murakami, H. et al. (2000) Effects of growing leafy daikon (Raphanus sativus) on populations of Plasmodiophora brassicae (clubroot). Plant Pathology, 49, 584-589.

6. Murakami, H. et al. (2001) Reduction of spore density of Plasmodiophora brassicae in soil by decoy plants. J. Gen. Plant Pathol., 67, 85-88.

7. Murakami, H., Tsushima, S. \& Shishido, Y. (2002) Factors affecting the pattern of the dose response curve of clubroot disease caused by Plasmodiophora brassicae. Soil Sci. Plant Nutr., 48, 421-427.

8. Naiki, T. (1987) Life cycle and control of Plasmodiophora brassicae, causing clubroot disease of cruciferous plants. Tsuchi to biseibutsu (Soil Microorganisms), 29, 25-39 [In Japanese with English summary].

9. Voorrips, R. E. (1996) A one-hit model for the infection of clubroot-susceptible cabbage (Brassica oleracea var. capitata) by Plasmodiophora brassicae at various inoculum densities. Euro. J. Plant Pathol., 102, 109-114.

10. Wallenhammar, A. C. (1996) Prevalence of Plasmodiophora brassicae in a spring oilseed rape growing area in central Sweden and factors influencing soil infestation levels. Plant Pathology, 45, 710-719.

11. Yamamura, K. \& Nemoto, H. (2003) Sampling plan using grade of infestation to estimate population density. Appl. Entomol. Zool., 38, 149-156. 